\title{
THE UNDETECTED ERROR PROBABILITY FOR REED-SOLOMON CODES
}

\author{
Kar-Ming Chcung Rabert J. McEliece \\ Communications Systems Research Section \\ Jet Propulsion Laboratory
}

\section{ABSTRACT}

This paper is an cxtension of a recont paper by McEliece and Suanson dealing with the decoder error probability for ReedSolomon codes (more gencrally, linfar MDS codes). McEliece and Swanson offered an upper bound on $P_{E}(u)$, the decoder error probability given $u$ symbol errors occur. In this paper, by using combinatoric technique like the principle of inclusion and exclusion, an exact formula for $P_{E}(u)$ is derined. The $P_{E}(u)$ of an MDS codc is obscrued to approach $Q$ rapidly as $u$ gets large, where $Q$ is the probability that a completely random error pattcrn will cause decoder crror. An upper bound for the expression $\mid \frac{P_{E}(11)}{-1}-1$ is derived. and is shoun to decrease nearly exponentially as $u$ increases. This proncs analytically that $P_{E}(u)$ indeed approaches $Q$ as $u$ becomes large, and some laus of large number come into play somchow.

\section{PART I}

\section{WEIGHT DISTRIBUTION FORMULA FOR DECODABLE WORDS IN AN MDS CODE}

\section{I.1. Introduction}

We begin with the following definitions. Let $C$ be a linear code of length $n$, dimension $k$, and minimum distance $d$. Let $q$ be a positive power of a prime. A $(n, k, d)$ linear code $C$ over $G F(q)$ is maximum distance separable (MDS) if the Singleton bound is achieved; that is, $d=n-k+1$. A code is $t$-error correcting if for some integer $t, 2 t \leq d-1$.

The class of Reed-Solomon (RS) codes is a subclass of the MDS code. Reed-Solomon codes are used in many sectors of today's industry. Some examples are the (255.223) 16-error correcting RS code (the NASA code) in deep space communications, the $(31,15) 8$-error correcting RS code (the JTIDS code ) in military communicalions, and the Cyclic Interleaving RS Code (CIRC) in compact disc industry. A detailed treat-

This work was supported in part by the National Aeronattics and Space Administration under Grant NAS7-918 and in part by the Air Force Office of Scicntific Research under Grant AFOSR-83-0296. ment of MDS codes, their properties and open questions about them is given in [1]. The weight distribulion of a linear MDS code with the parameters $n, k, d, t$, and $q$ was independently found by three groups of researchers: Assmus, Mattson and Turyn[2], Forney[3] and Kasami, Lin and Peterson[4].

In PART I by using the principle of inclusion and exclusion, we obtain the exact weight distribution formula for "decodable words" in any linear MIDS corle. By decodable words. we mean all the words that are lying within distance $t$ from a codeword. If we assume the decoder to be a bounded distance decoder, then the weight distribution formula for the decodable words can be used to find the undetected error probability for linear MDS codes. This will be discussed in detail in PART II.

\section{I.2. Derivation of For'mulae}

In this section, we introduce the basic tools that are required to derive the weight distribution formula for the number of decodable words in a linear MDS code. One important tool that we need is the basic combinatoric property of the MDS code. Let $k$ be a subset of $k$ coordinate positions of an MDS code. Let $\alpha=\left(a_{1}, a_{2}, \ldots, a_{k}\right)$ be a b-tuple of elements from $G F(q)$. By the basic combinatoric property of the MDS code [1], there exists a unique codeword whose $k$ coordinates in $K$ equal the $k$ components in $\alpha$. Another important tool that we need is the principle of inclusion and exclusion [5]. Suppose we have $N$ objects and a number of properties $P(1), \ldots, P(n)$. Let $N_{i}$ be the number of objects with property $P(i)$, and $N_{i_{1}, i_{2}, \ldots, i_{r}}$ be the number of objects with properties $P\left(i_{1}\right), P\left(i_{2}\right), \ldots$ $P\left(i_{r}\right)$. The number of objects $N(0)$ with none of the properties is given by the following formula [5]:

$$
\begin{aligned}
N(0)= & N-\sum_{i} N_{i}+\sum_{i_{1}<i_{2}} N_{i_{1} i_{2}}+\ldots+(-1)^{r} \\
& \sum_{i_{1}<i_{2} \ldots<i_{r}} N_{i_{1} i_{2} \ldots i_{r}}+\ldots+(-1)^{n} N_{123 \ldots n} .
\end{aligned}
$$

Using the mathematical tools mentioned above, $A_{u}$, the number of codeword of weight $u$ in a linear MDS code, can be easily derived [8]. This is given by the following expression:

(2) $\quad A_{u}=\left(\begin{array}{l}n \\ u\end{array}\right) \sum_{j=0}^{u-d}(-1)^{j}\left(\begin{array}{l}u \\ j\end{array}\right)\left(q^{u-d-j+1}-1\right) \quad d \leq u \leq n$. 
In this section, we derive a general formula for the number of decodable words of weiglit $u$, and we simplify the key equation by using some combinatoric identity.

Let $D$ be the set of decodable words in an MDS code. Let $V$ be a set of $v$ coordinates, $|V|=v$. Let $\left\{i_{1}, i_{2}, \ldots, i_{j}\right\}$ be a set of $j$ coordinates, where $\left\{i_{1}, i_{2}, \ldots, i_{j}\right\} \subset\{0,1,2 \ldots, n-1\}-V$. Define $S\left(i_{1}, i_{2}, \ldots, i_{j}\right)=\{d: \bar{d} \in D$ and $d$ has zeros in $V$ and $\left.\left\{i_{1}, i_{2}, \ldots, i_{j}\right\}\right\}$. We proceed to derive the weight distribution formula for the number of decodable words of weight $u$ in a linear MDS code by using the principle of inclusion and exclusion. Our problem is reduced to finding the cardinality of $S\left(i_{1}, i_{2}, \ldots, i_{j}\right)$ for all $j$ subjected to a given $V$. This problem is solved with the help of the following theorems.

Theorem J.1:

$$
\begin{aligned}
& \quad\left|S\left(i_{1}, i_{2}, \ldots, i_{j}\right)\right|=q^{u-d+1-j} V_{n}(t) \quad 0 \leq j \leq u-d \\
& \text { where } V_{n}(t)=\sum_{i=0}^{t}\left(\begin{array}{l}
n \\
i
\end{array}\right)(q-1)^{i}
\end{aligned}
$$$$
\text { Proof: }
$$

We note that each coset of a linear MDS code is also an MDS code. Also, since all words lying within the Ilamming spheres (with volume $V_{n}(t)$ ) that surround codewords are decodable words, we have $V_{n}(t)$ disjoint cosets that contain decodable words. From the basic combinatorial property of the MDS code we can, for each particular choice of $\left\{i_{1}, i_{2}, \ldots, i_{j}\right\}$, specify $q^{k \sim v-j}=q^{u-d+1-j}$ decodable words to each of these cosets. Thus, we have altogether $q^{u-d+1-j} V_{n}(t)$ decodable words having zeros at $V$ and $\left\{i_{1}, i_{2}, \ldots, i_{j}\right\}$. This completes the proof.

\section{Theorem I.2}

(4)

$\left|S\left(i_{1}, i_{2}, \ldots, i_{j}\right)\right|$

$$
\begin{gathered}
=\sum_{w=d-u+j}^{t}\left(\begin{array}{c}
n-u+j \\
w
\end{array}\right) \sum_{i=0}^{w-d+u-j}(-1)^{i}\left(\begin{array}{c}
w \\
i
\end{array}\right)\left(q^{w-d+u-j-i+1}-1\right) \\
\times \sum_{s=w}^{t}\left(\begin{array}{c}
u-j \\
s-w
\end{array}\right)(q-1)^{s-w}+\sum_{i=0}^{t}\left(\begin{array}{c}
u-j \\
i
\end{array}\right)(q-1)^{i}
\end{gathered}
$$$$
\text { for } u-d+1 \leq j \leq u-d+t
$$

Proof :

For $u-d+1=k-v \leq j$, the number of zeros in a decodable word is equal to $v+j \geq k$. Since $\tilde{d}$ is a decodable word, $\bar{d}$ can be uniquely decomposed into a codeword $\bar{c}$ and an error pattern $\bar{e}$ with weight that is less than or equal to $t$. If we "project" $\bar{c}$ onto $V \cup\left\{i_{1}, i_{2}, \ldots, i_{j}\right\}$, then the result will be a certain $(v+j, k)$ code. Since the parent code has a minimum distance $d=n-k+1$, the new code must have a mininum distance $d^{\prime} \geq d-(n-v-j)=(v+j)-k+1$. Since it is impossible for $d^{\prime}$ of the $(v+j, k)$ code to be greater than $(v+j)-k+1$ (because of the Singleton bound), $d^{\prime}$ must be equal to $d-(n-v-j)=(v+j)-k+1$.

If $\bar{c}+\bar{e}$ vanishes on $V \cup\left\{i_{1}, i_{2}, \ldots, i_{j}\right\}$, then $\bar{c}$ must have weight that is less than or equal to $t$ on $V \cup\left\{i_{1}, i_{2}, \ldots, i_{j}\right\}$. Let $w$ be the weight of $\bar{c}$ on $V \cup\left\{i_{1}, i_{2}, \ldots, i_{j}\right\}$. From the above argument we also know that $C$, when restricted to $V U$ $\left\{i_{1}, i_{2}, \ldots, i_{j}\right\}$, is a linear $(v+j, k)$ MDS code with a minimun distance $d-(n-v-j)=(v+j)-k+1$. Thus, $w$ is either 0 (in the case of the all-zero codeword) or is between $d-u+j$ and $t$. So the number of codewords of weight $w$ in the $(v+j, k)$ MDS code is (by using Equation (2))

$$
\left(\begin{array}{c}
n-u+j \\
w
\end{array}\right) \sum_{i=0}^{w-(d-(u-j))}(-1)^{i}\left(\begin{array}{c}
u \\
i
\end{array}\right)\left(q^{w-\{d-(u-j))-i+1}-1\right)
$$

for $d-u+j \leq w \leq t$ and 1 for $u=0$. For each codeword $\bar{c}$ with weight $w$ in $V \cup\left\{i_{1}, i_{2}, \ldots, i_{j}\right\}$, where $d^{\prime} \leq w \leq t\left(d^{\prime}=\right.$ $v+j-k+1)$, we must count the number of $\vec{e}$ 's such that $\bar{c}+\bar{e}$ vanishes on $V \cup\left\{i_{1}, i_{2}, \ldots, i_{j}\right\}$. Suppose that $\bar{e}$ has weight. $s \geq u \cdot \bar{e}$ must match $\bar{r}$ exactly on $V \cup\left\{i_{1}, i_{2}, \ldots, i_{j}\right\}$, but the $s-w$ other nonzero components can be arbitrarily placed outside $V \cup\left\{i_{1}, i_{2}, \ldots, i_{j}\right\}$. Then the total number of $\bar{e}$ 's for a given $\bar{c}$ of weight $w$ on $V \cup\left\{i_{1}, i_{2}, \ldots, i_{j}\right\}$ is $\sum_{s=w}^{t}\left(\begin{array}{c}u-j \\ s-u\end{array}\right)(q-$ $1)^{s-w}$. When $w=0$, all components of $\bar{c}$ must lie outside the set $V \cup\left\{i_{1}, i_{2}, \ldots, i_{j}\right\}$. So there are $\sum_{i=0}^{t}\left({ }^{u-j} i\right)(q-1)^{i} \bar{e}$ 's for the case $w=0$. Combining the above results, we obtain the theorem.

Theorem I.3:

$$
\left|S\left(i_{1}, i_{2}, \ldots, i_{j}\right)\right|=\sum_{i=0}^{t}\left(\begin{array}{c}
u-j \\
i
\end{array}\right)(q-1)^{i}
$$

for $u-d+t+1 \leq j \leq u-t-1$

Proof:

For $k-v+t \leq j \leq u-t-1$, the number of zeros in a decodable word is greater than or equal to $k+1$ but less than or equal to $n-t-1$. Thus any decodable words in $S\left(i_{1}, i_{2}, \ldots, i_{j}\right)$ have weight that is less than or equal to $d-t-1$. It is not hard to see that the element of $S\left(i_{1}, i_{2}, \ldots, i_{j}\right)$ cannot be decoded into a codeword of weight other than $\overrightarrow{0}$. Therefore, $S\left(i_{1}, i_{2}, \ldots, i_{j}\right)$ contains all words of weight that is less than or equal to $t$ in the coordinates $\{0,1, \ldots n-1\}-\left(V \cup\left\{i_{1}, i_{2}, \ldots, i_{j}\right\}\right)$. This completes the proof.

Theorem I.4:

$$
\left|S\left(i_{1}, i_{2}, \ldots, i_{j}\right)\right|=q^{u-j} \quad u-t \leq j \leq u
$$

Proof :

Since $j$ is greater than or equal to $u-t$, the number of zeros is equal to $v+j$ and is greater than or equal to $n-t$. Therefore, the number of nonzero components is less than or equal to $t$. Thus, all words with zeros on $V \cup\left\{i_{1}, i_{2}, \ldots, i_{j}\right\}$ are decodable and this completes the proof.

As in Part a, we choose $i_{1}, i_{2}, \ldots, i_{j}$ from $v=n-u$ coordinates. Thus, for every choice of $j$, we have $\left(\begin{array}{c}u \\ j\end{array}\right) S\left(i_{1}, i_{2}, \ldots, i_{j}\right)$ 's. Denote $N_{j}=\left(\begin{array}{l}u \\ j\end{array}\right)\left|S\left(i_{1}, i_{2}, \ldots, i_{j}\right)\right|$. Again, by the principle of inclusion and exclusion, we see that the number of decodable words which have exactly $v=n-u$ zeros at $V$ equals $\sum_{j=0}^{u}(-1)^{j} N_{j}$. However. we have $\left(\begin{array}{l}n \\ u\end{array}\right)=\left(\begin{array}{l}n \\ v\end{array}\right)$ ways to choose $v$ zeros from $0,1, \ldots, n-1$. Thus, the number of decodable words of weight $u$ is given by 
(7)

$$
D_{u}=\left(\begin{array}{l}
n \\
u
\end{array}\right) \sum_{j=0}^{u}(-1)^{j} N_{j} \quad \text { for } \quad d-t \leq u \leq n
$$

The weight enumerator formula that we have just derived is complicated and clumsy. There are four different expressions for $N_{j}$ 's, and these expressions are combined together by the inclusion and exclusion formula. The following theorem will show that the weight distribution formula for the number of decodable words in a linear MDS code can be simplified, and there are only two expressions for the $N_{j}$ 's.

Theorem 1.5:

$$
\begin{aligned}
A & =\sum_{j=0}^{u-t-1}(-1)^{j}\left(\begin{array}{l}
u \\
j
\end{array}\right) \sum_{i=0}^{t}\left(\begin{array}{c}
u-j \\
i
\end{array}\right)(q-1)^{i} \\
& +\sum_{j=u-t}^{u}(-1)^{j}\left(\begin{array}{l}
u \\
j
\end{array}\right) q^{u-j}=0
\end{aligned}
$$

Proof: see [8].

With Theorem $I .5$ and equation (3), (4), (5), (6) and (7), the weight enumerator formula can be simplified as follows :

(9) $D_{u}=\left(\begin{array}{l}n \\ u\end{array}\right) \sum_{j=0}^{u-d+t}(-1)^{j} N_{j} \quad$ for $\quad d-t \leq u \leq n$

(10) $N_{j}=\left(\begin{array}{l}u \\ j\end{array}\right)\left[q^{u-d+1-j} V_{n}(t)-\sum_{i=0}^{t}\left(\begin{array}{c}u-j \\ i\end{array}\right)(q-1)^{i}\right]$

$$
\text { for } 0 \leq j \leq u-d
$$

(11) $N_{j}=\left(\begin{array}{l}u \\ j\end{array}\right)\left[\sum_{w=d-u+j}^{t}\left(\begin{array}{c}n-u+j \\ w\end{array}\right)\right.$

$$
\begin{gathered}
\sum_{i=0}^{w-d+u-j}(-1)^{i}\left(\begin{array}{c}
w \\
i
\end{array}\right)\left(q^{w-d+u-j-i+1}-1\right) \\
\left.\sum_{s=w}^{t}\left(\begin{array}{c}
u-j \\
s-w
\end{array}\right)(q-1)^{s-w}\right] \\
\text { for } u-d+1 \leq j \leq u-d+t
\end{gathered}
$$

Examples of two simple linear MDS codes are given in table I.1 and table I.2.

\section{PART II}

\section{DECODER ERROR PROBABILITY OF A LINEAR MDS CODE}

\section{II.1. The Relationship Between $D_{u}$ And $P_{E}(u)$}

Let $C$ be an $(n, k, d)$ linear code capable of correcting $t$ errors. When a codeword $\bar{c} \in C$ is transmitted over a communication channel, channel noise may corrupt the transmitted signals. As a result, the receiver receives the corrupted version of the transmitted codeword $\bar{c}+\bar{e}$, where $\bar{e}$ is an error pattern of some weight $u$. If $u \leq t$, then a bounded distance decoder on the receiver's end detects and corrects the error $\bar{e}$ and recovers $\bar{c}$. If $u>t$, then the decoder fails and it either

i) detects the presence of the error pattern but is unable to correct it, or

ii) misinterprets (miscorrects) the received pattern $\bar{c}+\bar{e}$ for some other codeword $\vec{c}^{\prime}$ if the received pattern falls into the radius $t$ Hamming sphere of $\bar{c}^{\prime}$.

Case (ii) is, in most cases, more serious than case (i). This can occur (with a nonzero probability) when an error pattern $\bar{e}$ is of weight $u \geq d-t$. Let us further assume that all error patterns of weight $u$ are equally probable, and let us use $P_{E}(u)$ [7] to denote the decoder error probability given that an error pattern of weight $u$ occurs. It is not hard to see that $P_{E}(u)$ is given by the following expression:

$$
P_{E}(u)=\frac{D_{u}}{\left(\begin{array}{l}
n \\
u
\end{array}\right)(q-1)^{u}} \quad d-t \leq u \leq n .
$$

That is, $P_{E}(u)$ is the ratio of the number of decodable words of weight $u$ to the number of words of weight $u$ in the whole vector space. Thus, the problem of finding the $P_{E}(u)$ 's is essentially the same as the problem of finding the weight distribution of the set of decodable words. Equations (9), (10) and (11) of PART I and Equation (12) of PART II together enable us to find the exact decoder error probability of a linear MDS code.

Let the probability that a completely random error pattern will cause decoder error be denoted by $Q$. It is the ratio of the number of decodable words to the cardinality of the whole vector space. That is,

$$
Q=\frac{\left(q^{k}-1\right) V_{n}(t)}{q^{n}} \simeq q^{-(n-k)} V_{n}(t),
$$

where $n-k$ is the code's redundancy and $V_{n}(t)=\sum_{i=0}^{t}\left(\begin{array}{c}n \\ i\end{array}\right)(q-$ $1)^{i}$ is the volume of a Hamming sphere of radius $t$. It is shown in the next section that if $q \geq n$, which is generally true, then $P_{E}(u)$ approaches $Q$ very rapidly as $u$ increases. 


\section{II.2. Examples and Observations}

Two well-known examples of linear MDS codes - the NASA code and the JTIDS code - are tabulated in Table II.1 and Table II.2, respectively. From the above two examples, we observe that $P_{E}(u)$ approaches the constant $Q$ as $u$ increases. In fact, $P_{E}(u)$ approaches $Q$ rapidly for $u \ll n$. In the case of large $q$ and $q \geq n, P_{E}(u)$ approaches $Q$ even for $u<d$. The $P_{E}(u)$ and $Q$ of the NASA code agree to eight significant digits for $u \geq 26(d=33)$. If $P_{E}(u)$ and $Q$ are interpreted combinatorically as ratios, then we have the following relationship:

$$
\begin{aligned}
& \frac{\text { \# of decodable words of weight } u}{\text { \# of vectors of weight u }} \\
& \rightarrow \frac{\text { \# of decodable words }}{\text { \# of words in vector space }}
\end{aligned}
$$

This astonishing relationship cited above implies that a linear MDS code, which possesses rigid algebraic and combinatori structures, behaves (in some sense) like a random code with no structure at all. Some laws of large number come into play somehow. In order to describe analytically how fast $P_{E}(u)$ approaches $Q$ when $u$ is large, an upper bound on the expression $\left|\frac{P_{E}(u)}{Q}-1\right|$ is derived in [8]. This upper bound is denoted by $\mathrm{U}(u)$, where $u \geq d$, and is given by the following expression $[8]$ :

$$
\begin{aligned}
\left|\frac{P_{E}(u)}{Q}-1\right| & \leq\left(\frac{q}{q-1}\right)^{t-1} \frac{q^{d-1}\left(\begin{array}{c}
u \\
d-1
\end{array}\right) d}{(q-1)^{u}}+\frac{q^{d-1} 2^{u}}{(q-1)^{u}} \frac{V_{u}^{*}(t)}{V_{n}(t)} \\
& =\mathrm{U}(u),
\end{aligned}
$$

where $V_{u}^{*}(t)=\sum_{i=0}^{t}\left(\begin{array}{c}u \\ i\end{array}\right)\left(\frac{q-1}{2}\right)^{i}$. It is shown that $\mathrm{U}(u)$ approaches a very small number $\epsilon$ as $u$ increases. The upper bounds of $\left|\frac{P E(u)}{Q}-1\right|$ of the NASA code and the JTIDS code are tabulated in Table II.3 and Table II.4 respectively.

Example $1(4,2)$ MDS code over $G F(5)$ with $t=1$.

\begin{tabular}{c} 
Weight \\
\hline 0 \\
1 \\
2 \\
3 \\
4
\end{tabular}

\begin{tabular}{l} 
\# of decodable words \\
\hline 1 \\
16 \\
48 \\
192 \\
168
\end{tabular}

Total number of decodable words $=q^{k} V_{n}(t)=425$.

Table I.1
With the assumptions that $q$ is greater tharr or equal to $n$ and $u$ is large compared to $d$, Equation (14) shows that the upper bound of $\left|\frac{P_{E}(u)}{Q}-1\right|$ is dominated by the denominator term $(q-1)^{u}$. Thus, the upper bound of $\left|\frac{P_{E(u)}}{Q}-1\right|$ decays nearly exponentially as a function of $u$. This upper bound is not a very tight bound, but it is sufficient to illustrate the point that $P_{E}(u)$ approaches $Q$ very rapidly as $u$ increases.

\section{REFERENCES}

[1] F. J. MacWilliams and N. J. A. Sloane, The Theory of Error-Correcting Codes. Amsterdam, The Netherlands: North-Holland, 1983.

[2] Assmus,E. F., H. F. Matton, Jr., and R. J. Turyn, Cyclic Codes, AFCRL-65-332, Air Force Cambridge Research Labs, Bedford, Mass., 1965.

[3] Forney, G. D., Jr., Concatenated Codes, The M.I.T. Press, Cambridge, Mass., 1966.

[4] Kasami, T., S. Lin, and W. W. Peterson, "Some Results on Weight Distributuons of BCH codes," IEEE Trans. Inform. Theory, IT-12 (1966): 274.

[5] Marshall Hall, Combinatorial Theory, Blaisdell, 1967.

[6] E. R. Berlekamp and J. L. Ramsey, 1978 "Readable erasures improve the performance of Reed-Solomon codes," IEEE Tran. Inform. Theory, vol. IT-24, 632-633

[7] R. J. McEliece and L. Swanson, 1986 "On the Decoder Error Probability for Reed-Solomon Codes," IEEE Tran. Inform. Theory, vol. IT-32, 701-703.

[8] K-M. Cheung, "On the Error-Correction Schemes of Data Storage Systems", Ph.D. Thesis, 1987. 
Example $2(6,3)$ MISS code over $G F(4)$ with $t=1$.

\begin{tabular}{c} 
Wrigin \\
\hline 0 \\
1 \\
2 \\
3 \\
4 \\
5 \\
6
\end{tabular}

\# of decodable words
1
18
0
180
405
378
234

\begin{tabular}{l} 
Inper bound][6] \\
\hline- \\
- \\
- \\
180 \\
855 \\
1026 \\
513
\end{tabular}

Total number of decodabie words $=q^{k} V_{n}(t)=1216$.

Table I.2

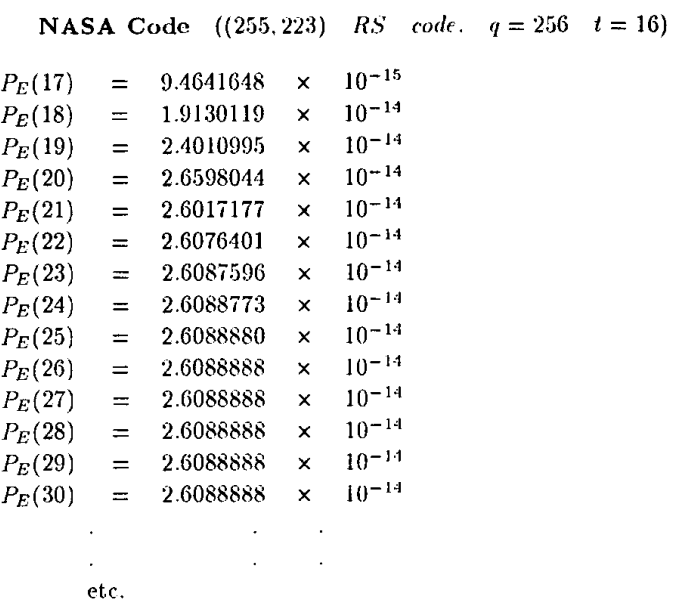

JTIDS Code $((31,15)$ RS code. $\varphi=32 \quad t=8)$

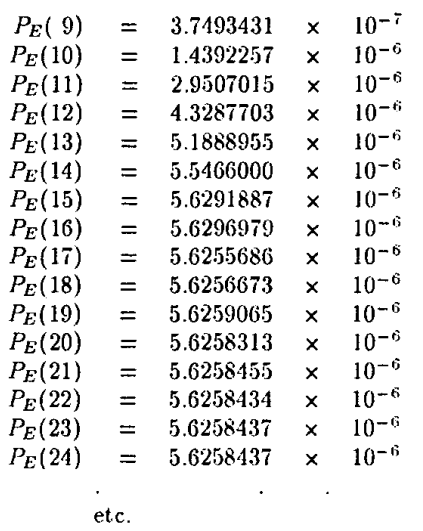

Table II.2

Table II.1

NASA Code $(255,223)$ RS Ciode. $q=256 \quad t=16$

$\begin{array}{cllllll}u: & 33 & 34 & 35 & 36 & 37 & \ldots \\ \mathrm{U}(u): & 5.133 & 0.3422 & 0.0157 & 5.526 \times 10^{-4} & 1.512 \times 10^{-5} & \ldots\end{array}$

Table II.3

$\begin{array}{cllllll}\text { JTIDS Code } & (31,15) & \text { RS } & \text { Code. } & q=32 \quad t=8 \\ & & & & \\ u: & 17 & 18 & 19 & 20 & 21 & \ldots \\ \mathrm{U}(u): & 19.35 & 5.618 & 1.148 & 0.1851 & 0.02508 & \ldots\end{array}$

Table II.4 